\title{
Assessment of Prescribing and Monitoring Habits for Patients Taking an Antiarrhythmic and Concomitant QTc-Prolonging Antibiotic
}

\author{
Kelsey Noss, Sandra M. Aguero and Travis Reinaker* \\ Einstein Medical Center-Philadelphia, 5501 Old York Road, Philadelphia, PA 19141, USA; \\ kelsey.noss12@gmail.com (K.N.); aguersan@einstein.edu (S.M.A.) \\ * Correspondence: reinaket@einstein.edu; Tel.: +1-570-881-0432
}

Received: 26 September 2017; Accepted: 30 October 2017; Published: 1 November 2017

\begin{abstract}
Patients may intermittently require antimicrobial therapy with a QTc-prolonging antibiotic, which presents a challenge for prescribers of patients already taking a QTc-prolonging antiarrhythmic. Manufacturers recommend close monitoring for evidence of QTc-prolongation with the concomitant use of QTc-prolonging medications, but the monitoring parameters are not well-defined. Previous studies recommend a surveillance electrocardiogram (EKG) be completed both before and after the initiation of QTc-prolonging medications, but it is unknown to what degree EKGs displaying the QTc-interval are used to alter physician order entry and pharmacist order verification during concomitant therapy. A retrospective chart review was conducted between October 2015-September 2016 to assess prescribing and monitoring habits for patients taking an antiarrhythmic and a concomitant QTc-prolonging antibiotic. Of the 42 patients who received at least one dose of two QTc-prolonging agents, 36 (85.7\%) received a baseline EKG, and $23(63.8 \%)$ received a follow-up EKG. Pharmacists intervened on this drug-drug interaction and recommended follow-up EKGs only three times $(8.3 \%)$ and offered alternative therapy recommendations once $(2.8 \%)$. The QTc-interval was not optimally monitored in some instances for patients concomitantly receiving two QTc-prolonging agents. These results stress the importance of inter-professional communication to place an emphasis on follow-up monitoring or use of alternative therapy agents to avoid the drug-drug interaction altogether.
\end{abstract}

Keywords: QTc-prolongation; electrocardiogram; antiarrhythmic; macrolide; fluoroquinolone; drug-interaction

\section{Introduction}

The QT-interval on an electrocardiogram (EKG) represents the depolarization and repolarization of cardiac ventricles. On a 12-lead EKG, the QT-interval is measured from the beginning of the QRS complex to the end of the $\mathrm{T}$ wave as it returns to baseline. Several factors such as gender, heart rate, underlying arrhythmias, and conduction defects influence the QT-interval. The QT-interval will vary depending on heart rate. To standardize the measurement to a heart rate of 60 beats per minute, the QT-interval is corrected and referred to as the QTc [1]. The QTc allows for comparison of the QT-interval across a range of heart rates. The most universally adopted method for correcting QT-intervals for heart rate is the Bazett's formula (Corrected QT-interval (QTc): QT $/ \sqrt{ } / R R$ in seconds; RR is the interval from the peak of one QRS complex to the peak of the next as shown on an electrocardiogram) [1]. QTc-prolongation is defined as a QTc-interval of $>450$ milliseconds (ms) in males, and $>470 \mathrm{~ms}$ in females, and can predispose patients to life-threatening ventricular arrhythmias. Several medications have been implicated in the prolongation of the QTc-interval. A complete resource of medications, stratified according to QTc-prolonging risk, can be found at crediblemeds.com. 
QTc-prolongation can occur in up to 10\% of patients taking QTc-prolonging antiarrhythmics (including amiodarone), and $<1 \%$ of patients taking macrolide or fluoroquinolone antibiotics. The concomitant use of two QTc-prolonging medications increases this risk [1]. Manufacturers recommend close monitoring for evidence of QTc-prolongation with the concomitant use of QTc-prolonging medications, while previous studies recommend that offending drugs should be discontinued in patients who develop a prolonged QTc-interval $>500 \mathrm{~ms}$, or an increase in QTc-interval of $>60 \mathrm{~ms}$ on follow-up EKG [2-8]. However, it is unknown whether EKGs are used to alter prescribing and monitoring habits when these medications are combined. This is the first study to observe the real-life prescribing and monitoring habits for patients taking an antiarrhythmic and a concomitant QTc-prolonging antibiotic at a large, academic medical center.

\section{Materials and Methods}

A retrospective chart review, exempt from IRB-approval, was completed to observe the prescribing and monitoring habits for patients taking an antiarrhythmic and a concomitant QTc-prolonging antibiotic. This study included patients admitted to Einstein Medical Center-Philadelphia from 1 October 2015 to 30 September 2016. Patients were identified with an electronic report of drug interaction alerts that had advised pharmacists during order verification of the increased risk of a QTc-prolonging effect between two medications. Patients taking amiodarone upon admission who also received at least one concomitant dose of ciprofloxacin, moxifloxacin, or azithromycin during admission were included. Gender, QTc-prolonging medication, medication dose, pharmacist interventions, presence or absence of a baseline (while on amiodarone only) and follow-up (while on amiodarone and a QTc-prolonging antibiotic), QTc-interval, change in QTc-interval, and therapy modification and justification were collected. The formula used to calculate the corrected QT-interval was Bazett's formula. Descriptive statistics, including the median and range, were used to analyze patient demographics, prescriptions, and monitoring data.

\section{Results}

A total of 78 patients were assessed, and 42 patients received concomitant QTc-prolonging agents. The most commonly prescribed medication regimen was azithromycin added to home amiodarone therapy in 23 patients (54.5\%). Thirty-six out of 42 patients $(85.7 \%)$ previously taking amiodarone received a baseline EKG (Table 1). The median male QTc-interval was $473 \mathrm{~ms}$ (range: 405-602 ms), and the median female QTc-interval was $470 \mathrm{~ms}$ (range: 435-599 ms) (Table 1). Of the male patients who received a baseline EKG, nine out of $14(64.2 \%)$ had a prolonged QTc-interval ( $>450 \mathrm{~ms})$. Of the female patients who received a baseline EKG, 12 out of $22(54.5 \%)$ had a prolonged QTc-interval ( $>470 \mathrm{~ms}$ ) (Table 1). Of the 36 patients who received a baseline EKG, a pharmacist recommended a follow-up EKG on three occasions (8.3\%) (Table 1). Twenty-three out of 36 patients (63.8\%) received a follow-up EKG. The median male QTc-interval was $481 \mathrm{~ms}$ (range: 440-628 ms), and the median female QTc-interval was $484 \mathrm{~ms}$ (range: 384-645 ms) (Table 1). Of the male patients who received a follow-up EKG, two out of eight $(25 \%)$ had a prolonged QTc-interval $(>450 \mathrm{~ms})$. Of the female patients who received a follow-up EKG, 11 out of $15(73.3 \%)$ had a prolonged QTc-interval (>470 ms) (Table 1). Ten out of 23 patients $(43.5 \%$ ) had a QTc-interval $>500 \mathrm{~ms}$ or an increase in QTc-interval of $>60 \mathrm{~ms}$ on follow-up EKG, but in only three out of 23 instances (13\%), therapy was either discontinued or a different antimicrobial was utilized (Table 1). Of these three patients, two (66.7\%) experienced a QTc-interval increase to $>600 \mathrm{~ms}$ without arrhythmia, and one (33.3\%) developed torsades de pointes before alternative therapy was utilized. (Table 1 ). 
Table 1. Electrocardiogram (EKG) results and subsequent therapy modifications. QTc: corrected QT-interval.

\begin{tabular}{cc}
\hline Baseline EKG obtained (\%) & $36 / 42(85.7)$ \\
Median male QTc, ms (range) & $473(405-602)$ \\
Male QTc >450 ms (\%) & $9 / 14(62.4 \%)$ \\
Median female QTc, ms (range) & $470(435-599)$ \\
Female QTc $>470$ ms (\%) & $12 / 22(54.5 \%)$ \\
\hline Follow-up EKG recommended by pharmacist (\%) & $3 / 36(8.3)$ \\
Follow-up EKG obtained (\%) & $23 / 36(63.8)$ \\
Median male QTc, ms (range) & $481(440-628)$ \\
Male QTc $>450$ ms (\%) & $2 / 8(25)$ \\
Median female QTc, ms (range) & $484(384-645)$ \\
Female QTc $>470$ ms (\%) & $11 / 15(73.3)$ \\
\hline Follow-up EKG QTc-interval >500 ms, or QTc-interval increase of $>60$ ms $(\%)$ & $10 / 23(43.5)$ \\
Therapy changed based on follow-up EKG $(\%)$ & $3 / 23(13)$ \\
Patient developed torsades de pointes $(\%)$ & $1 / 23(4.3)$ \\
Patient QTc-interval increased to $>600$ ms $(\%)$ & $2 / 23(8.7)$ \\
\hline
\end{tabular}

\section{Discussion}

Manufacturers recommend close monitoring for evidence of QTc-prolongation with the concomitant use of QTc-prolonging medications, but the monitoring parameters are not well-defined [2-5]. Previous studies have recommended that surveillance EKGs be completed before and after initiation of QTc-prolonging medications [1,6-8]. This observational study demonstrated that despite prescribers ordering baseline EKGs on most patients, $36.2 \%$ of patients still did not receive a follow-up EKG. In the presence of a follow-up EKG, "The Significance of QT-Interval in Drug Development" published in the British Journal of Clinical Pharmacology states that offending drugs should be discontinued in patients who develop an increase of $>60 \mathrm{~ms}$ in QTc-interval [7]. Additionally, "Practice Standards for Electrocardiographic Monitoring in Hospital Setting" published by the American Heart Association (AHA) states that offending drugs should be discontinued in patients who develop a prolonged QTc-interval $>500 \mathrm{~ms}$ [8]. In our study, ten patients demonstrated either a QTc-interval $>500 \mathrm{~ms}$, or an increase of $>60 \mathrm{~ms}$ in QTC-interval on follow-up EKG. Prescribers infrequently responded with therapy modifications, which may have led to one patient experiencing torsades de pointes. To avoid the potential development of fatal arrhythmias in the setting of a prolonged QTc-interval, alternative antibiotics could be utilized. The Infectious Disease Society of America provides alternative recommendations for specific disease states, and an antibiotic that does not prolong the QTc-interval could be selected. Pharmacists infrequently recommended follow-up monitoring or offered alternative treatment recommendations. Lack of documentation may have led to the perceived small amount of pharmacist interventions. Pharmacists were also not able to view follow-up EKGs, as results are not readily reposted in the electronic medical record. Additional limitations to the study include its small sample size, and other medications or patient-specific characteristics that cause QTc-prolongation were not assessed. Our study showed that the QTc-interval was not optimally monitored in some instances for patients, despite recommendations from manufacturers. For instance, six patients did not receive a baseline EKG, and 13 patients did not receive a follow-up EKG. These results may also be experienced in other institutions, stressing the importance of inter-professional communication to place an emphasis on follow-up monitoring or use of alternative therapy agents to avoid the drug-drug interaction altogether.

Author Contributions: Kelsey Noss, Sandra M. Aguero, and Travis Reinaker conceived and designed the experiments; Experiments were not performed as this was a retrospective chart review; Kelsey Noss and Travis Reinaker analyzed the data; Kelsey Noss and Travis Reinaker contributed reagents/materials/analysis tools; Kelsey Noss and Travis Reinaker wrote the paper.

Conflicts of Interest: Authors report no conflicts of interest. 


\section{References}

1. Nachimuthu, S.; Schussler, J.M. Drug-induced QT interval prolongation: Mechanisms and clinical management. Ther. Adv. Drug Saf. 2012, 3, 241-253. [CrossRef] [PubMed]

2. Amiodarone [package insert]. Sagent Pharmaceuticals, Inc.: Schaumbrug, IL, USA, July 2011. Available online: http:/ / www.sagentpharma.com/wp-content/uploads/2014/11/Amiodarone_PI1.pdf (accessed on 15 May 2017).

3. Ciprofloxacin [package insert]. Bayer HealthCare Pharmaceuticals, Inc.: Wayne, NJ, USA, February 2009. Available online: https:/ / www.accessdata.fda.gov/drugsatfda_docs/label/2009/019537s073,020780s030lbl. pdf (accessed on 15 May 2017).

4. Moxifloxacin [package insert]. Bayer Pharmaceuticals, Inc.: West Haven, CT, USA, December 1999. Available online: https:/ / www.accessdata.fda.gov /drugsatfda_docs/label/1999/21085lbl.pdf (accessed on 15 May 2017).

5. Azithromycin [package insert]. Pfizer Labs, Inc.: New York, NY, USA, January 2013. Available online: https:/ / www.accessdata.fda.gov/drugsatfda_docs/label/2013/050710s039,050711s036,050784s023lbl.pdf (accessed on 15 May 2017).

6. Barbara, J.B.; Ackerman, M.J.; Funk, M.; Gibler, B.W.; Kligfield, P.; Menon, V.; Philippides, G.J.; Roden, D.M.; Wojciech, Z. Prevention of torsade de pointes in hospital settings: A scientific statement from the American Heart Association and the American College of Cardiology Foundation. Circulation 2010, 121, 1047-1060. [CrossRef]

7. Shah, R.R. The significance of QT interval in drug development. Br. J. Clin. Pharmacol. 2002, 54, 188-202. [CrossRef] [PubMed]

8. Drew, B.J.; Califf, R.M.; Funk, M.; Kaufman, E.S.; Krucoff, M.W.; Laks, M.M.; Macfarlane, P.W.; Sommargren, C.; Swiryn, S.; Van Hare, G.F. Practice standards for electrocardiographic monitoring in hospital settings: An American Heart Association scientific statement from the councils on cardiovascular nursing, clinical cardiology, and cardiovascular disease in the young: Endorsed by the international society of computerized electrocardiology and the American Association of Critical-Care Nurses. Circulation 2004, 110, 2721-2746. [CrossRef] [PubMed] 